\title{
Distribuição de abundância de espécies da comunidade arbórea do topo de um morro na floresta estacional subtropical
}

\author{
Species abundance distribution in tree community of \\ a hill top in subtropical seasonal forest
}

\author{
Alessandro Abreu Fávero ${ }^{\mathrm{I}^{*}}$ Malcon do Prado Costa ${ }^{\mathrm{II}}$ Maurício Figueira ${ }^{\mathrm{I}}$ \\ Daiane Deckmann Andriollo ${ }^{\mathrm{I}}$ Solon Jonas Longhi ${ }^{\mathrm{II}}$
}

\section{RESUMO}

Modelos ecológicos de distribuição de espécies (DAEs) em uma comunidade arbórea foram investigados para constatar: (i) se as variáveis ambientais são divididas de maneira estocástica e de modo similar entre as espécies; (ii) se cada espécie exaure mais da metade do nicho ecológico existente; (iii) se a comunidade arbórea possui muitas espécies com níveis intermediários de abundância e poucas espécies raras e comuns; (iv) e se, na sucessão secundária, as espécies colonizadoras tardias têm as maiores necessidades de um nicho especializado, de tal modo que são mais raras que as espécies colonizadoras iniciais. Obteve-se os dados em oito unidades amostrais de dimensões de $20 \mathrm{~m} \times 50 \mathrm{~m}$, distribuidas sistematicamente, perfazendo 0,8ha de amostra; ajustaram-se cinco modelos de DAEs (broken-stick, niche-preemption, log-normal, Zipf e Zipf-Mandelbrot) para a amostra e para as unidades amostrais; os critérios de acurácia dos modelos foram: Akaike, Bayesiano e Deviance. O modelo ecológico de DAEs que descreveu o ranking/abundância das espécies para a comunidade arbórea foi o Zipf-Mandelbrot. Os modelos ecológicos de DAEs que caracterizaram as unidades amostrais foram: niche-preemption, Zipf e Zipf-Mandelbrot. O modelo ZipfMandelbrot postula a colonização das espécies sob as exigências de condições ambientais prévias para o estabelecimento, assim, detecta-se uma comunidade arbórea em estágio de maturidade. Os modelos ecológicos de DAEs obtidos para as unidades amostrais, separadamente, evidenciam a disparidade da distribuição de abundância de espécies no interior da comunidade arbórea, que se interpretou como o efeito da heterogeneidade das condições e dos recursos ambientais, além de perturbações naturais, que originam um mosaico vegetacional.

Palavras-chave: árvores, curvas de Whittaker, diversidade biológica, dominância ecológica, ecologia florestal.

\begin{abstract}
Ecological Models of Species abundance distribution (SADs) of a tree community were investigated to find: (i) if environmental variables are divided stochastically and similarly between species; (ii) if each species exhausts more than half of the existing ecological niche; (iii) if the tree community has many species with intermediate levels of abundance and a few rare and common species; (iv) and if, in secondary succession, the later colonizing species have greatest needs for a specialized niche, so, they are more rare than the initial colonizers species. The data were obtained in eight sampling units with dimensions of $20 \mathrm{~m}$ x 50m, distributed in a systematic way, totaling 0.8 ha sample; grouped in five models of SADs (broken-stick, niche-preemption, lognormal, Zipf and Zipf-Mandelbrot) for the sample of the tree community and the eight sampling units; the criteria of accuracy of the models were: Akaike, Bayesian and Deviance. The ecological model described the SADs that rank/abundance of tree species for ecological community which was the Zipf-Mandelbrot. The ecological models of SADs that characterized the eight sampling units were niche-preemption, Zipf and Zipf-Mandelbrot. The ZipfMandelbrot model postulates the colonization of species under the requirements of environmental preconditions for the establishment of the species, and thus detects up an arboreal community stage of maturity. The ecological models of SADs obtained for the sampling units, separately, show the disparity in the distribution of species abundance within the tree community that was interpreted as the effect of heterogeneity of conditions and environmental resources, and natural disturbances, originating a mosaic vegetation.
\end{abstract}

Key words: trees, curves Whittaker, biological diversity, ecological dominance, forest ecology.

\section{INTRODUÇÃO}

Nas comunidades ecológicas, as espécies não possuem a mesma abundância. $\mathrm{O}$ que ocorre

\footnotetext{
'Programa de Pós-graduação em Engenharia Florestal, Universidade Federal de Santa Maria (UFSM), 97105-900, Santa Maria, RS, Brasil. E-mail: abreufavero@yahoo.com.br.*Autor para correspondência.

IIPrograma de Pós-graduação em Ciências Florestais, Universidade Federal do Rio Grande do Norte (UFRN), Macaíba, RN, Brasil.

IIIPrograma de Pós-graduação em Engenharia Florestal, Universidade Federal de Santa Maria (UFSM), Santa Maria, RS, Brasil.
} 
universalmente é que poucas espécies são abundantes, outras moderadamente comuns e o restante, a maioria, são raras (MAGURRAN, 2006), sendo à dominância numérica de poucas espécies uma das leis gerais da ecologia (MC GILL et al., 2007). A abundância de espécie reflete a variedade e o arranjo de condições e recursos ecológicos disponíveis para a população, bem como as influências dos competidores, predadores e doenças (TOWNSEND et al., 2010).

As DAES são modelos ecológicos da abundância de diferentes espécies encontradas nas comunidades biológicas (MAGURRAN, 2006; OKSANEN et al., 2010). Para ser preciso, é definido como vetor de abundância das espécies presentes (MC GILL et al., 2007) e são descritas por modelos probabilísticos (PRADO, 2009). Atualmente, existem 40 modelos classificados em cinco famílias: purely statical, branching process, population dynamics, niche partitioning e spatial distribuition of individuals. Estes modelos ecológicos permitem detectar padrões de dominância, raridade e uniformidade e testam hipóteses sobre a organização das espécies nas comunidades ecológicas (MAGURRAN, 1988), possibilitado compreenderem as causas da raridade (HILL et al., 1998) e dos distúrbios nos ecossistemas naturais (HUBBEL, 2001).

As suposições investigadas dos modelos ecológicos de DAEs são examinadas de acordo com as premissas dos seguintes modelos: brokenstick (McARTHUR, 1957), niche-preemption (MOTOMURA, 1932), log-normal (PRESTON, 1948), Zipf (ZIPF, 1949) e Zipf-Mandelbrot (GRAY, 1987). O modelo broken-stick fornece evidências de que alguma variável ambiental está sendo dividida de maneira estocástica e similar entre as espécies de uma comunidade ecológica, é usado como hipótese nula contra a qual outros padrões de divisão de nicho podem ser testados (MAGURRAN, 2006). $\mathrm{O}$ modelo niche-preemption assume que cada espécie exaure mais da metade do espaço de nicho existente, descreve as comunidades ecológicas menos uniformes (ODUM, 1988). O modelo log-normal prediz uma comunidade ecológica com muitas espécies com níveis intermediários de abundância e poucas espécies raras e comuns (RICKLEFS, 1993). Os modelos Zipf e Zipf-Mandelbrot são interpretados como o processo sucessional no qual espécies colonizadoras tardias têm as maiores necessidades de um nicho especializado e por isso são mais raras que as espécies colonizadoras iniciais (MAGURRAN, 2006), postulam que a presença de uma espécie devese a dependências de condições físicas e ecológicas prévias. As espécies pioneiras possuem baixo custo ecológico, sendo pouco exigentes, enquanto as espécies tardias têm o custo ecológico elevado de energia, tempo e organização do ecossistema antes de se estabelecerem (WILSON, 1991). O modelo Zipf possui os parâmetros: $\mathrm{p}_{1}$ - proporção ajustada da espécie mais abundante; $\gamma$ - coeficiente de decaimento; o modelo Zipf-Mandelbrot acrescenta o parâmetro $\mathrm{p}_{2}$ $(\beta)$, para o modelo Zipf, e substitui $p_{1}$ por $\mathrm{c}$ - constante do escalonamento (OKSANEN et al., 2010).

Devido à heterogeneidade de varáveis ambientais e ecológicas na estrutura de uma comunidade arbórea, as plantas distribuem-se ao longo de gradientes e em mosaicos de vegetação, podendo, deste modo, formar grupos de ranking/ abundância de espécies. Nesse contexto, a presente pesquisa caracteriza os modelos ecológicos de DAEs de uma comunidade arbórea situada no topo de um morro, "Morro do Elefante", na Floresta Estacional Subtropical no sul do Brasil.

\section{MATERIAL E MÉTODOS}

Local de estudo - o estudo ocorreu na Floresta Latifoliada Estacional Subtropical Submontana de Cumeada (OLIVEIRA-FILHO; 2009), no topo de um morro conhecida como "Morro do Elefante", $29^{\circ} 40^{\prime}$ 'S e $53^{\circ} 43^{\prime} \mathrm{W}$, a cerca de $460 \mathrm{~m}$ de altitude sobre o nível do mar, situada na região central do rebordo do Planalto Meridional, no estado do Rio Grande do sul, Brasil.

Coleta dos dados - a obtenção dos dados ocorreu no topo da montanha, com área de 11,5ha, onde foram instaladas oito unidades amostrais com dimensões de $20 \mathrm{~m}$ x $50 \mathrm{~m}$, distribuídas de forma sistemática, distantes a $50 \mathrm{~m}$ entre si, perfazendo 0,8 ha de amostragem.

Nas parcelas, foram identificados os indivíduos com CAP (Circunferência à Altura do Peito, à altura de $1,30 \mathrm{~m}$ do solo) $\geq 15,7 \mathrm{~cm}$, conforme os estudos realizados em florestas subtropicais do Brasil. As espécies foram identificadas in loco e das dúbias coletou-se o material botânico. A classificação das famílias botânicas foi conforme Angiosperm Phylogeny Group (APG III, 2009) e dos gêneros e das espécies, segundo SOBRAL et. al. (2013) e a Lista de Espécies da Flora do Brasil.

Análise dos dados - cinco modelos ecológicos de DAEs (Tabela 1) foram aplicados para caracterizar e avaliar a comunidade arbórea como um todo e, nas oito unidades amostrais, separadamente, para detectar a heterogeneidade da riqueza e da abundância das espécies.

Os modelos ecológicos de DAEs ajustados e mais adequados foram escolhidos usando a 
Tabela 1 - Modelos e equações de SADs aplicados para a comunidade florestal do morro do Elefante; âr = abundância de espécie esperada no rank $\mathrm{r}$; $\mathrm{S}=$ número de espécies; $\mathrm{N}=$ número dos indivíduos; $\Phi$ $=$ função normal padrão; $\mathrm{p}_{1}=$ proporção estimada das espécies mais abundantes; $r=$ rank da espécie; $\mathrm{k}, \mu, \gamma, \beta$ e $\mathrm{c}=$ parâmetros que são estimados para cada modelo.

\begin{tabular}{ll}
\hline Modelos & Equações \\
\hline Broken-stick & $\hat{a} r=\frac{N}{S} \sum_{k=r}^{S} \frac{1}{k}$ \\
Preemption & $\hat{a} r=N \alpha(1-\alpha)^{r-1}$ \\
Lognormal & $\hat{a} r=\exp [\log (\mu)+\log (\beta) \Phi]$ \\
Zipf & $\hat{a} r=N p_{1} r^{\gamma}$ \\
Zipf-Mandelbrot & $\hat{a r}=N c(r+\beta)^{\gamma}$ \\
\hline
\end{tabular}

estimativa da probabilidade máxima, que compara os modelos através do critério de informação de Akaike (AIC), Bayesian (BIC) e Deviance (resíduos do ajuste do modelo), os menores valores de $A I C$, $B I C$ e Deviance indicam o melhor modelo ecológico (OKSANEN et al., 2010).

Para o processamento dos dados, utilizouse o pacote vegan v1.17.9 (OKSANEN et al., 2010) do sofware R v2.11.1, (R Development Core Team, 2010).

\section{RESULTADOS}

Registrou-se 1496 árvores, distribuídas em 57 espécies, 46 gêneros e 27 famílias. As famílias botânicas com maior riqueza de espécies foram Fabaceae e Lauraceae (5 spp.) e Meliaceae e Myrtaceae (4 spp.), juntas representam 31,57\% do total de espécies. As famílias botânicas com maior abundância foram Euphorbiaceae (320 indivíduos) e Moraceae (289) seguidas de Lauraceae (161), Meliaceae (157), Sapindaceae (108), Myrtaceae (84) e Primulaceae (82), perfazendo 80,28\% das árvores. As espécies mais abundantes foram Sorocea bonplandii (Baill.) W.C. Burger et al.(18,92\% dos indivíduos) e Actinostemon concolor (Spreng.) Müll. Arg. (14,24\%), seguidas de Trichilia clausenii C. DC. (7,09\%), Alchornea triplinervea (Spreng.) Müll. Arg. (6,89\%) e Cupania vernalis Cambess. (5,78\%); dez espécies foram representadas por apenas um indivíduo, correspondendo a $17,54 \%$ do total das espécies e $0,67 \%$ do total das árvores.
$\mathrm{Na}$ comunidade arbórea, ocorrem duas espécies dominantes $(33,16 \%$ indivíduos $), 19$ espécies com abundância intermediária $(59,02 \%)$ e 36 espécies com a abundância baixa $(7,82 \%)$, menos de dez indivíduos. O modelo ecológico de DAEs que melhor descreveu a comunidade ecológica como um todo foi o Zipf-Mandelbrot (Figura 1). Os gráficos de ranking/abundância das espécies nas oito unidades amostrais (Figura 1) estão constituídos, basicamente, por duas espécies dominantes, sendo que as espécies $\boldsymbol{S}$. bonplandii $\mathrm{A}$. concolor ocorrem (dominantes) na maioria das unidades amostrais.

De modo geral, as oito unidades amostrais são representadas por duas espécies dominantes, poucas espécies com a abundância intermediária e as demais espécies, a maioria, com a abundância baixa (menos de dez indivíduos). A tabela 2 compara os modelos ecológicos de DAEs usando o critério de informação de $A I C$ e $B I C$, os menores valores indicam o melhor modelo.

Dos cinco modelos ecológicos de DAEs que foram usados para caracterizar as oito unidades amostrais ocorreram apenas três (Figura 1): nichepreemption, Zipf e Zipf-Mandelbrot. A unidade amostral número cinco ajustou-se ao modelo Zipf, cuja curva do modelo foi a mais longa (maior riqueza de espécies) e menos inclinada (maior uniformidade). E a unidade amostral número dois apresentou o modelo geométrico, indicando a baixa uniformidade. Dentre as unidades amostrais ajustadas ao modelo Zipf-Mandelbrot com a maior riqueza de espécies e uniformidade, foi a número sete que possui a curva do modelo ecológico de DAEs mais longa e menos inclinada.

\section{DISCUSSÃO}

O número de árvores registradas na comunidade arbórea é de moderado a elevado, comparado a outras pesquisas na região (MACHADO \& LONGHI, 1990; FARIAS et al., 1994; LONGHI et al., 2000; BUDKE et al., 2004; LINDENMAIER et al., 2006; GIEHL et al., 2007), ressaltando que existem diferenças nos critérios de inclusão destes trabalhos e considerando as condições do local de estudo, tais como, solo raso, ventos fortes, efeito de borda, entre outros.

As famílias botânicas Fabaceae, Lauraceae, Meliaceae e Myrtaceae foram as mais importantes na riqueza de espécies. Dentre estas, a Fabaceae possui o modo de dispersão anemocórica (MORELATTO, 1992; ALBERTI, 2002), que são favorecidas pelos ventos fortes no topo da montanha, e as demais são zoocóricas, demonstrando que a comunidade arbórea do topo do "Morro do Elefante" 


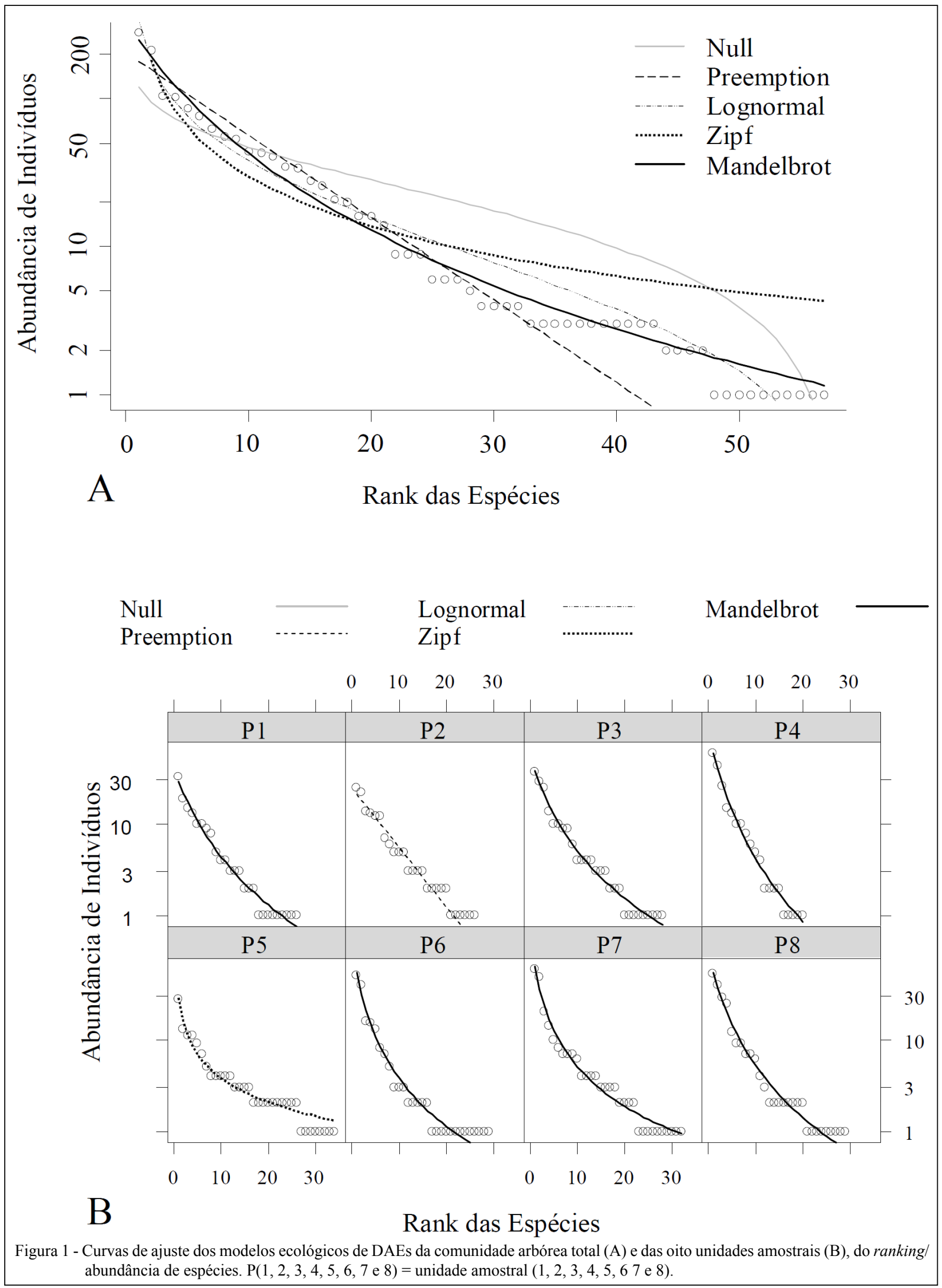

Ciência Rural, v.45, n.5, mai, 2015. 
Tabela 2 - Modelos ecológicos de DAEs e critério de informação $A I C$ e $B I C$ das oito unidades amostrais instaladas na comunidade arbórea; valores em negrito indicando os menores valores de $A I C$ e $B I C ; \mathrm{P}(1,2,3,4,5,6,7$ e 8) = unidade amostral $(1,2,3,4,5,6,7$ e 8); par = parâmetro. M-1 = NullModel, M-2 = Preemption, M-3 = Lognormal, M-4 = Zipf, M-5 = Zipf-Mandelbrot.

\begin{tabular}{|c|c|c|c|c|c|c|c|c|c|c|c|}
\hline$P 1$ & M-1 & M-2 & M-3 & M-4 & M-5 & $P 2$ & M-1 & M-2 & M-3 & M-4 & M-5 \\
\hline par1 & & 0,15 & 1,18 & 0,26 & 96,16 & par1 & & 0,14 & 1,29 & 0,23 & 1038,70 \\
\hline par2 & & & 1,15 & $-0,99$ & $-2,79$ & par2 & & & 1,03 & $-0,90$ & $-3,31$ \\
\hline par3 & & & & & 8,25 & par3 & & & & & 13,07 \\
\hline Deviance & 14,10 & 7,29 & 5,14 & 12,19 & 3,12 & Deviance & 8,78 & 4,87 & 6,35 & 14,63 & 2,41 \\
\hline$A I C$ & 94,34 & 89,53 & 89,38 & 96,43 & 89,36 & $A I C$ & 91,30 & 89,39 & 92,87 & 101,15 & 90,93 \\
\hline$B I C$ & 94,34 & 90,78 & 91,89 & 98,94 & 93,13 & $B I C$ & 91,30 & 90,65 & 95,38 & 103,66 & 94,71 \\
\hline P3 & M-1 & M-2 & M-3 & M-4 & M-5 & P4 & M-1 & M-2 & M-3 & M-4 & M-5 \\
\hline par1 & & 0,16 & 1,24 & 0,27 & 29,99 & par1 & & 0,23 & 1,56 & 0,35 & 175,40 \\
\hline par2 & & & 1,21 & $-1,03$ & $-2,51$ & par2 & & & 1,36 & $-1,20$ & $-3,27$ \\
\hline par3 & & & & & 6,32 & par3 & & & & & 6,15 \\
\hline Deviance & 25,74 & 12,22 & 9,31 & 17,28 & 3,59 & Deviance & 39,16 & 10,37 & 8,15 & 18,56 & 3,46 \\
\hline$A I C$ & 113,92 & 102,40 & 101,48 & 109,45 & 97,76 & $A I C$ & 107,89 & 81,10 & 80,88 & 91,29 & 78,19 \\
\hline$B I C$ & 113,92 & 103,73 & 104,15 & 112,12 & 101,76 & $B I C$ & 107,89 & 82,09 & 82,87 & 93,28 & 81,17 \\
\hline$P 5$ & M-1 & M-2 & M-3 & M-4 & M-5 & P6 & M-1 & M-2 & M-3 & M-4 & M-5 \\
\hline par1 & & 0,10 & 0,97 & 0,20 & 0,37 & par1 & & 0,19 & 0,86 & 0,34 & 3,21 \\
\hline par2 & & & 1,00 & $-0,87$ & $-1,08$ & par2 & & & 1,50 & $-1,24$ & $-2,02$ \\
\hline par3 & & & & & 0,97 & par3 & & & & & 2,28 \\
\hline Deviance & 15,40 & 17,62 & 5,42 & 3,93 & 3,12 & Deviance & 74,28 & 36,19 & 18,27 & 15,02 & 6,38 \\
\hline AIC & 115,24 & 119,45 & 109,26 & 107,76 & 108,95 & $A I C$ & 158,43 & 122,34 & 106,42 & 103,17 & 96,53 \\
\hline$B I C$ & 115,24 & 120,97 & 112,31 & 110,82 & 113,53 & $B I C$ & 158,43 & 123,70 & 109,15 & 105,90 & 100,63 \\
\hline$P 7$ & M-1 & M-2 & M-3 & M-4 & M-5 & $P 8$ & M-1 & M-2 & M-3 & M-4 & M-5 \\
\hline par1 & & 0,16 & 1,07 & 0,31 & 0,93 & parl & & 0,18 & 1,17 & 0,31 & 22,78 \\
\hline par2 & & & 1,42 & $-1,17$ & $-1,55$ & par2 & & & 1,40 & $-1,15$ & $-2,55$ \\
\hline par3 & & & & & 11973,00 & par3 & & & & & 4,93 \\
\hline Deviance & 75,31 & 51,28 & 16,96 & 12,89 & 8,39 & Deviance & 62,65 & 23,88 & 17,53 & 24,21 & 5,22 \\
\hline$A I C$ & 174,9 & 152,9 & 120,6 & 116,5 & 114,01 & $A I C$ & 153,1 & 116,3 & 111,9 & 118,6 & 101,61 \\
\hline$B I C$ & 174,9 & 154,4 & 123,5 & 119,4 & 118,41 & $B I C$ & 153,1 & 117,6 & 114,7 & 121,3 & 105,71 \\
\hline Modelos & & \multicolumn{2}{|c|}{ M-1 } & \multicolumn{2}{|c|}{ M-2 } & \multicolumn{2}{|c|}{ M-3 } & \multicolumn{2}{|c|}{ M-4 } & \multicolumn{2}{|c|}{ M-5 } \\
\hline par1 & & & & & & & & \multicolumn{2}{|c|}{$\mathrm{M}-4$} & \multicolumn{2}{|c|}{268,63} \\
\hline par2 & & & & & & \multicolumn{2}{|c|}{21,26} & \multicolumn{2}{|c|}{0,26} & \multicolumn{2}{|c|}{$\begin{array}{l}200,00 \\
-30,29\end{array}$} \\
\hline par3 & & & & \multicolumn{2}{|c|}{0,12} & \multicolumn{2}{|c|}{15,70} & \multicolumn{2}{|c|}{$-11,17$} & \multicolumn{2}{|c|}{10,46} \\
\hline Deviance & & \multicolumn{2}{|c|}{617,37} & \multicolumn{2}{|c|}{142,83} & \multicolumn{2}{|c|}{75,85} & \multicolumn{2}{|c|}{239,11} & \multicolumn{2}{|c|}{49,61} \\
\hline$A I C$ & & \multicolumn{2}{|c|}{839,52} & \multicolumn{2}{|c|}{366,98} & \multirow{2}{*}{\multicolumn{2}{|c|}{$\begin{array}{l}302,00 \\
306,09\end{array}$}} & \multicolumn{2}{|c|}{465,26} & \multicolumn{2}{|c|}{277,77} \\
\hline$B I C$ & & \multicolumn{2}{|c|}{839,52} & \multicolumn{2}{|c|}{369,02} & & & & & & \\
\hline
\end{tabular}

constitui um local de oferta de recursos e condições ecológicas paraa fauna, sobretudoparaasaves(DELLA FLORA, 2010) e os morcegos (PATHEK et al., 2007).

A fisionomia da comunidade arbórea é caracterizada principalmente por espécies arbóreas de abundância intermediária. As famílias botânicas Euphorbiaceae, Moraceae e Meliaceae são, respectivamente, as de maior número de árvores, sendo representadas pelas respectivas espécies, $A$. concolor, S. bonplandii e $\boldsymbol{T}$. claussenii, árvores de pequenos portes, ficando no sub-bosque da floresta. Por outro lado, as espécies com poucos indivíduos caracterizam a fisionomia da comunidade arbórea, devido as suas dimensões e às características de suas copas específicas no dossel ou como emergentes, destas, destacamse: Schefflera calva (Cham.) Frodin \& Fiaschi, Ficus cestrifolia Schott, Myrocarpus frondosus Allemão, Parapiptadenia rigida (Benth.) Brenan, Nectandra lanceolata Ness, Cordia trichotoma (L.) Gottschling \& J. E. Mill. e Cedrela fissilis Vell.. Em relação às espécies com apenas um indivíduo, que contribuem na estrutura fisionômica da floresta, destaca-sequenão são espécies denaturezarara no domínio fitogeográfico (REITZ et al., 1988) e sim espécies de baixa densidade na comunidade arbórea, quando do momento do inventário no local de estudo, por serem espécies pioneiras e estas são frequentes em clareiras e bordas da comunidade arbórea. 
O ranking/abundância das espécies da comunidade arbórea detectou a dominância de espécies que são típicas do sub-bosque, constituídas de pouca biomassa, essas são esciófitas, o que indica que, para se desenvolverem, exigem condições específicas geradas pelas árvores do dossel e emergentes, indicando um fragmento florestal com maturidade e perturbações pretéritas.

$\mathrm{Na}$ comunidade arbórea estudada, existe a dominância ecológica de duas espécies, de forma hierárquica, $\boldsymbol{S}$. bonplandii, que ocupa as porções mais elevadas do sub-bosque, e $\boldsymbol{A}$. concolor, que ocupa as porções mais baixas, de tal modo que partilham recursos e condições ambientais similares.

Assim, os dados estudados foram ajustados no modelo Zipf-Mandelbrot, não devido à maior abundância de espécies pioneiras, mas devido à presença de poucas espécies dominantes no subbosque, o que caracteriza um grau de maturidade, na maioria das zonas do mosaico da comunidade arbórea. Apesar de o modelo Zipf-Mandelbrot partir do princípio de que as espécies pioneiras, de maior abundância em comunidades vegetais tropicais, carecem de sítios específicos para a colonização das espécies mais exigentes em sombra, a interpretação deste modelo é diferente, devido à ocorrência do estudo ser em uma Floresta Estacional Subtropical.

O modelo ecológico de DAEs ajustado para a comunidade arbórea, Zipf-Mandelbrot, corrobora a interpretação acima, que a comunidade arbórea possui espécies que são exigentes às condições ecológicas proporcionadas por espécies pioneiras e clímax dependente de luz (SWAINE \& WHITMORE, 1988), sendo essas consideradas como facilitadoras da sucessão ecológica secundária.

Essa característica denota, também, a rusticidade das espécies esciófitas para o nicho que ocupam, pois os indivíduos de suas populações são os mais aptos a sobreviverem à competição, devido a certos caracteres de sua história evolutiva, que lhe permitem transpor barreiras restritivas - os filtros -, impostas pelo ambiente sombreado. Sendo assim, suas populações compõem a maior porção da comunidade vegetal do sub-bosque da floresta, e os mecanismos de dispersão, colonização, ocupação e estabilização das espécies arbóreas no topo do "Morro do Elefante" se enquadra nos pressupostos de assembleias ecológicas (KEDDY \& WEITHER, 2001).

$\mathrm{O}$ ranking/abundância das espécies nas oito unidades amostrais seguiu o mesmo padrão da comunidade arbórea como um todo, com duas espécies dominantes, poucas espécies com abundância intermediária e um número relativamente alto de espécies de baixa densidade populacional. Todavia, entre as unidades amostrais, ocorreu a variação na composição florística e na riqueza de espécies, repercutindo em diferentes modelos ecológicos de DAEs ajustados, o que caracteriza a heterogeneidade espacial no interior da comunidade arbórea.

Dentre as oito unidades amostrais a DAEs da unidade amostral, a número dois foi ajustada ao modelo niche-preemption, o qual indica condições extremas (ODUM, 1988), é encontrada em ambientes pobres em espécies, em estágios iniciais de sucessão secundária (MAGURRAN, 2006), as espécies dominantes são heliófitas (REITZ et al., 1988) e se desenvolvem nas clareiras. Já a unidade amostral número cinco foi ajustada ao modelo Zipf, demonstrando que esta unidade amostral está pouco perturbada (MAGURRAN, 2006) e em estágio inicial de maturidade. Nas demais unidades amostrais, o modelo de DAEs foi o Zipf-Mandelbrot, entretanto, entre essas unidades amostrais, verificou-se uma variação na riqueza de espécies e na composição florística, devido à heterogeneidade das condições e recursos ambientais da comunidade arbórea, que diferenciaram os comprimentos e as inclinações de curvas dos modelos ecológicos de DAEs.

A variação na composição florística, na riqueza de espécies e nos modelos ecológicos de DAEs entre as unidades amostrais não podem ser creditadas somente à heterogeneidade ambiental, já que as unidades amostrais estão sujeitas a fatores edáficos, climáticos e topográficos similares. Sendo, portanto, provável que a heterogeneidade de modelos ecológicos de DAEs esteja associada ao espectro de fatores ecológicos, tais como, perturbações naturais e nas diferenças de sucesso na dispersão, colonização e estabelecimento das espécies, além dos efeitos dos gradientes de luz, ventos e umidade da borda para o centro da comunidade arbórea, no topo do morro.

Assim, a heterogeneidade da composição florística, da riqueza de espécies e de modelos ecológicos de DAEs ao longo de gradientes de recursos e de condições ambientais indica a heterogeneidade e/ou perturbações ecológicas no interior da comunidade arbórea, incidindo em diferentes ranking/abundância de espécies e modelos de ecológicos DAEs, permitindo detectar, deste modo, a ocorrência de zonas de mosaico vegetacional no interior da comunidade arbórea.

\section{CONCLUSÃO}

O modelo ecológico de DAEs ajustado para a comunidade arbórea foi o Zipf-Mandelbrot, 
que postula a colonização e as exigências de condições ambientais prévias para o estabelecimento de árvores de algumas espécies e, assim, detecta-se que a comunidade arbórea encontra-se em estágio de maturidade. A variação da composição florística, riqueza e abundância das espécies entre as oito unidades amostrais indica a heterogeneidade $\mathrm{e}$ perturbações ambientais no interior da comunidade ecológica, refletindo em diferentes ranking/ abundância de espécies e modelos ecológicos de DAEs no interior da comunidade arbórea, formando um mosaico vegetacional.

\section{REFERÊNCIAS}

ALBERTI, L.F. Fenologia de uma comunidade florestal em Santa Maria, RS. 2002. 144f. Dissertação (Mestrado em Engenharia Florestal) - Curso de Pós-graduação em Engenharia Florestal, Universidade Federal de Santa Maria, RS.

ANGIOSPERM PHYLOGENY GROUP III. An update of the Angiosperm Phylogeny Group classification for the orders and families of flowering plants: APG III. Botanical Journal of the Linnean Society, v.161, p.105-121, 2009. Disponível em: <http:// onlinelibrary.wiley.com/doi/10.1111/j.1095-8339.2009.00996.x/ pdf $>$. Acesso em: 20 jul. 2012. doi:10.1111/j.10958339.2009.00996.x.

BUDKE, J.C. et al. Florística e fitossociologia do componente arbóreo de uma floresta ribeirinha, arroio Passos das Tropas, Santa Maria, RS, Brasil. Acta botanica brasilica, v.18, n.3, p.581589, 2004. Disponível em: <http://dx.doi.org/10.1590/S010233062004000300016>. Acesso em: 14 mar. 2012. doi: 10.1590/ S0102-33062004000300016.

DELLA FLORA, F. Ecologia comportamental do tangara Chiroxiphilacaudata (aves, Pipridae) no extremo sul da floresta atlântica. 2010. 94f. Dissertação (Mestrado em Ciências Biológicas) - Curso de Pós-graduação em Biodiversidade Animal, Universidade Federal de Santa Maria, RS.

FARIAS, J.A.C. et al. Estrutura fitossociológica de uma floresta estacional decidual na região de Santa Maria, RS. Ciência Florestal, v.4, n.1, p.109-128. 1994. Disponível em: <http:// cascavel.ufsm.br/revistas/ojs-.2.2/index.php/cienciaflorestal/ article/download/301/168>. Acesso em: 12 abr. 2011. doi: $10.5902 / 19805098$

GIEHL, E.L.H. et al. Princípios de estatísticas em ecologia. Porto Alegre: Artmed. 2011. 528p.

GRAY, J.S. Species-abundance patterns. In: GEE, J.H.R.; GILLER, P.S. (EDS.). Organization of communities - past and present. Oxoford, UK: Blackwell Science, 1987. p.53-67.

HILL, J.K.; HAMER, K.C. Using species abundance models as indicators of habitat disturbance in tropical forest. British Ecological Society. Journal of Applied Ecology, v.35, n.3, p.458-460, 1998. Disponível em: <http://onlinelibrary.wiley.com/ doi/10.1046/j.1365-2664.1998.00310.x/pdf>. Acesso em: 2 abr. 2012. doi: 10.1046/j.1365-2664.1998.00310.x
HUBBELL, S.P. The unified neutral theory of biodiversity and biogeography. Princeton, New Jersey: Princeton University, 2001. 448p

KEDDY, P.; WEITHER, E. The scope and goals of research on assembly rules. Introduction. In: WEITHER, E.; KEDDY, P. (Eds.). Ecological assembly rules: perspectives, advances, retreats. Cambridge: Cambridge University, 2001. p.1-20.

LINDENMAIER, D.S.; BUDKE, J.C. Florística, diversidade e distribuição espacial das espécies arbóreas em uma floresta estacional na bacia do Rio Jacuí, sul do Brasil. Pesquisas Botância, n.57, p.193-216, 2006. (São Leopoldo, Instituto Anchietano de Pesquisa). Disponível em: <http://www.anchietano.unisinos.br/ publicacoes/botanica/botanica57/artigo9.pdf>. Acesso em: 20 abr. 2011. ISSN-0373-840 X

Lista de Espécies da Flora do Brasil. Jardim Botânico do Rio de Janeiro. Disponível em: <http://floradobrasil.jbrj.gov.br/>. Acesso em: 14 fev. 2014.

LONGHI, S.J. et al. Aspectos fitossociológicos de fragmento da floresta estacional decidual, Santa Maria, RS. Ciência Florestal, v.10, n.2, p.59-74, 2000. Disponível em: $<$ http://cascavel.ufsm. br/revistas/ojs-2.2.2/index.php/cienciaflorestal/article/view/471>. Acesso em: 2 fev. 2011. doi: 10.5902/19805098.

MACHADO, P.F.S.; LONGHI, S.J. Aspectos florísticos e fitossociológicos do Morro do Elefante, Santa Maria, RS. Revista Centro de Ciências Rurais, v.20, n.3-4, p.261-280, 1990. Disponível em: <http://coral.ufsm.br/revistaccr/index.php/RCCCR/ article/view/741>. Acesso em: 25 dez. 2011. ISSN: 0085-5901

MAGURRAN, A.E. Ecological diversity and its measurement. 2.ed. Princeton: Princeton University, 1988. 179p.

MAGURRAN, A.E. Measuring biological diversity. Maldem, MA: Blackwell Publishing, 2006. 256p.

MacARTHUR, R. On the relative abundance of bird species. Proceedings of the National Academy of Sciences of the United States of America, v.43, n.3, p.293-295, 1957. Disponível em: $<$ http://www.jstor.org/stable/89566>. Acesso em: 22 mar. 2011.

McGILL, B.J. et al. Fractals, form, change and dimension. San Francisco, California: W.H. Freeman, 1977. 365p.

MORELATTO, L.P.C. (Org.). História natural da Serra do Japi: ecologia e preservação de uma área florestal no sudeste do Brasil. Campinas: UNICAMP, 1992. 321p.

MOTOMURA, I. On the statistical treatment of communities. Zoological Magazine, v.44 p.379-383, 1932. [Impresso]

ODUM, E.P. Ecologia. Rio de Janeiro: Guanabara, 1988. 434p.

OKSANEN, J. et al. Vegan: community ecology package. R package version 1.17.9. 2010. Disponível em: $<$ http://CRAN.Rprojetct.Org/package=vegan $>$. Acesso em: 05/04/2012.

OLIVEIRA-FILHO, A.T. Classificação das fitofisionomias da América do Sul cisandina tropical e subtropical: proposta de um novo sistema prático e flexível ou uma injeção a mais de caos? Rodriguesia, v.60, n.2, p.237-258, 2009. Disponível em: <http:// www.jstor.org/discover/10.2307/23499986>. Acesso em: 23 mar. 2010. Doi: $10.2307 / 23499986$. 
PATHEK, D.B. et al. Micro-hábitat, horário de atividade, dieta e dispersão de sementes por morcegos do "morro do elefante", Santa Maria, RS, Brasil. In: CONGRESSO DE ECOLOGIA DO BRASIL, 8., 2007, Caxambu, MG. Anais... Caxambu: Sociedade Brasileira de Botânica, 2007. p. 1-2.

PRADO, P.I.K.L. Distribuição de abundância de espécies. Avanços analíticos para entender um padrão básico em ecologia. Ciência \& Ambiente, v.1, n.39, p. 121-136, 2009. [Impresso]

PRESTON, F.W. The commonness and rarity of species. Ecology, v.29, n.3, p.254-283, 1948.

REITZ, R. et al. Projeto madeira do Rio Grande do Sul. Porto Alegre: Sedesul, 1988. 525p.

RICKLEFS, R.E. A economia da natureza. 3.ed. Rio de Janeiro: Guanabara Koogan. 1996. 470p.
SOBRAL, M.; JARENKOW, J.A. Flora arbórea e arborescente do Rio Grande do Sul, Brasil. 2.ed. São Carlos: RiMa, 2013, 357p.

SWAINE, M.D.; WHITMORE, T.C. On the definition of ecological species groups in tropical rain forests. Vegetatio, v.75, n.1-2, p.81-86,1988. Disponível em: <http://link.springer.com/ article/10.1007\%2FBF00044629>. Acesso em: 23 mar. 2010. Doi: 10.1007/BF00044629

TOWNSEND, C.R. et al. Fundamentos em ecologia. 3.ed. Porto Alegre: Artmed, 2010. 576p.

WILSON, J.B. Methods for fitting dominance/diversity curves. Journal of Vegetation Science, v. 2, n. 1, p.35-46, 1991. Disponível em: $\quad<$ http://onlinelibrary.wiley.com/doi/10.2307/3235896/pdf $>$. Acesso em: 23 mar. 2010. Doi: 10.2307/3235896.

ZIPF, G.K. Human behavior and the principle of least effort. Cambridge, Massachusetts: Addison-Wesly, 1949. 573p. 


\section{ERRATA}

No artigo "Distribuição de abundância de espécies da comunidade arbórea do topo de uma montanha na floresta subtropical estacional decídua” publicado no fascículo v45n5 de maio da Ciência Rural na nota de rodapé que possui idenditificação institucional dos autores onde se lia:

“'IIIPrograma de Pós-graduação em Engenharia Florestal, Universidade Federal do Paraná (UFPR), Curitiba, PR, Brasil.”

leia-se:

"'IIIPrograma de Pós-graduação em Engenharia Florestal, Universidade Federal de Santa Maria (UFSM), Santa Maria, RS, Brasil."

Para a versão correta, acesse:

http://www.scielo.br/pdf/cr/v45n5/0103-8478-cr-45-05-00806.pdf 